\title{
Two-level corpectomy versus three-level discectomy for cervical spondylotic myelopathy: a comparison of perioperative, radiographic, and clinical outcomes
}

\author{
Darryl Lau, MD, Dean Chou, MD, and Praveen V. Mummaneni, MD \\ Department of Neurological Surgery, University of California, San Francisco, California
}

OBJECT In the treatment of cervical spondylotic myelopathy (CSM), anterior cervical corpectomy and fusion (ACCF) and anterior cervical discectomy and fusion (ACDF) are effective decompressive techniques. It remains to be determined whether ACCF and ACDF offer equivalent outcomes for multilevel CSM. In this study, the authors compared perioperative, radiographic, and clinical outcomes between 2-level ACCF and 3-level ACDF.

METHODS Between 2006 and 2012, all patients at the authors' hospital who underwent 2-level ACCF or 3-level ACDF performed by 1 of 2 surgeons were identified. Primary outcomes of interest were sagittal Cobb angle, adjacent-segment disease (ASD) requiring surgery, neck pain measured by visual analog scale (VAS), and Nurick score. Secondary outcomes of interest included estimated blood loss (EBL), length of stay, perioperative complications, and radiographic pseudarthrosis rate. Chi-square tests and 2-tailed Student t-tests were used to compare the 2 groups. A subgroup analysis of patients without posterior spinal fusion (PSF) was also performed.

RESULTS Twenty patients underwent 2-level ACCF, and 35 patients underwent 3-level ACDF during a 6 -year period. Preoperative Nurick scores were higher in the ACCF group (2.1 vs 1.1, $p=0.014$ ), and more patients underwent PSF in the 2-level ACCF group compared with patients in the 3-level ACDF group (60.0\% vs $17.1 \%, p=0.001)$. Otherwise there were no significant differences in demographics, comorbidities, and baseline clinical parameters between the 2 groups. Two-level ACCF was associated with significantly higher EBL compared with 3-level ACDF for the anterior stage of surgery ( $382.2 \mathrm{ml}$ vs $117.9 \mathrm{ml}, p<0.001)$. Two-level ACCF was also associated with a longer hospital stay compared with 3-level ACDF (7.2 days vs 4.9 days, $p=0.048$ ), but a subgroup comparison of patients without PSF showed no significant difference in length of stay (3.1 days vs 4.4 days for 2-level ACCF vs 3 -level ACDF, respectively; $p=0.267$ ). Similarly, there was a trend toward more complications in the 2-level ACCF group (20.0\%) than the 3-level ACDF group $(5.7 \% ; p=0.102)$, but a subgroup analysis that excluded those who had second-stage PSF no longer showed the same trend (2-level ACCF, $0.0 \%$ vs 3-level ACDF, $3.4 \% ; p=0.594$ ). There were no significant differences between the ACCF group and the ACDF group in terms of postoperative sagittal Cobb angle $\left(7.2^{\circ}\right.$ vs $\left.12.1^{\circ}, p=0.173\right)$, operative ASD $(6.3 \%$ vs $3.6 \%, p=0.682)$, and radiographic pseudarthrosis rate $(6.3 \%$ vs $7.1 \%, p=0.909)$. Both groups had similar improvement in mean VAS neck pain scores (3.4 vs 3.2 for ACCF vs ACDF, respectively; $p=0.860$ ) and Nurick scores $(0.8$ vs $0.7, p=0.925$ ).

CONCLUSIONS Two-level ACCF was associated with greater EBL and longer hospital stays when patients underwent a second-stage PSF. However, the length of stay was similar when patients underwent anterior-only decompression with either 2-level ACCF or 3-level ACDF. Furthermore, perioperative complication rates were similar in the 2 groups when patients underwent anterior decompression without PSF. Both groups obtained similar postoperative cervical lordosis, operative ASD rates, radiographic pseudarthrosis rates, neurological improvement, and pain relief.

http://thejns.org/doi/abs/10.3171/2014.12.SPINE14545

KEY WORDS adjacent-segment disease; corpectomy; cervical spondylotic myelopathy; discectomy; fusion; pseudarthrosis; sagittal curvature

\footnotetext{
ABBREVIATIONS ACCF = anterior cervical corpectomy and fusion; $\mathrm{ACDF}=$ anterior cervical discectomy and fusion; $\mathrm{ASD}=$ adjacent-segment disease; $\mathrm{CSM}=\mathrm{cervical}$ spondylotic myelopathy; EBL = estimated blood loss; PSF = posterior spinal fusion; VAS = visual analog scale.

SUBMITTED May 31, 2014. ACCEPTED December 3, 2014.

INCLUDE WHEN CITING Published online June 19, 2015; DOI: 10.3171/2014.12.SPINE14545.

DISCLOSURE Dr. Mummaneni receives royalties from DePuy Spine, Quality Medical Publishers, and Thieme Publishers; he receives honoraria from DePuy Spine, AOSpine, and Globus; and he has stock in Spinicity/ISD. Dr. Chou has received honoraria from Globus, Medtronic, DePuy, and Orthofix for teaching. No funding was provided for this study. The authors report no conflict of interest concerning the materials or methods used in this study or the findings specified in this paper.
} 
$\mathrm{C}$ ERVICAL spondylotic myelopathy (CSM) is typically found in patients over the age of 50 years..$^{22,26}$ Ongoing CSM can result in spinal cord compression and even injury., ${ }^{9,26}$ Fortunately, CSM can often be readily treated with surgical decompression, anterior column reconstruction, and fusion. ${ }^{15} \mathrm{An}$ anterior, posterior, or staged anterior-posterior approach can be taken depending on the clinical situation and the goal of surgery. When an anterior approach is taken, anterior cervical corpectomy and fusion (ACCF) and anterior cervical discectomy and fusion (ACDF) are the 2 main modalities of decompression, ${ }^{11,15}$ and both techniques have proven to be safe and effective.$^{23}$ However, CSM often involves multiple levels, and the surgical management of more extensive disease can be challenging.

The superiority of ACCF or ACDF for multilevel CSM remains controversial. ${ }^{9} \mathrm{ACDF}$ is an effective method to decompress the anterior spinal cord and preserve spinal stability, and the procedure is associated with a low prevalence of graft extrusion or migration. ${ }^{7}$ Some surgeons argue, however, that ACDF is not optimal for multilevel CSM because of the higher chance of incomplete decompression behind the midvertebral body, limited visual access during surgery, and higher risk of pseudarthrosis due to more graft-host interfaces (especially in the case of multilevel CSM). ${ }^{2,17}$ Alternatively, ACCF minimizes the number of graft-host interfaces, allows more extensive decompression, and provides a source for bony autograft to promote fusion. ${ }^{4}$ The drawbacks to ACCF are the potential for a higher incidence of complications and graft extrusion due to the fewer possible points for ventral plate screw fixation. ${ }^{24}$

Only a few studies have compared the use of the 2 decompressive techniques for the treatment of multilevel CSM that spans more than 2 disk levels. Therefore, the purpose of this study was to evaluate and compare the outcomes associated with 2-level ACCF and 3-level ACDF by assessing various perioperative, radiographic, and clinical outcomes.

\section{Methods}

This study was formally approved by the University of California, San Francisco, Committee on Human Research.

\section{Patients}

All patients over the age of 18 years who underwent ACCF or ACDF from 2006 to 2012 were identified. Records of patients who underwent either a 2-level ACCF or 3-level ACDF following a diagnosis of CSM were then extracted from the list, and surgical outcomes were evaluated. Clinical indications for surgery were neurological deficit, myelopathy, and/or intractable pain with associated imaging findings consistent with CSM. All surgical procedures were performed by 1 of 2 senior spine surgeons (P.V.M. or D.C.). The decision regarding whether ACCF or ACDF was to be performed as the method of anterior decompression was based on the following: if the pathology causing the compression was located mainly posterior to the center of the vertebral body, then ACCF was performed, but if the patient refused to have an allograft implanted (for cultural reasons, many of our Asian patients refused an allograft), then ACCF was performed to obtain local autograft material. The ACCF procedures were performed with the use of polyetheretherketone cages (Fig. 1 left) or expandable cages. The ACDF procedures were performed with the use of structural allograft or polyetheretherketone cages filled with local autograft or allograft material (Fig. 1 right). All patients underwent anterior plate fixation. The decision to perform posterior spinal fusion (PSF) was determined on a case-by-case basis based on intraoperative findings of osteopenia. It was not standard practice for patients to undergo dual-energy $\mathrm{x}$-ray absorptiometry scans. Rather, osteopenia was assessed by the quality of screw purchase at the screw and bone interface. If the screw-bone interface was weak, PSF was used. All PSF procedures were done via lateral mass screws and posterior lateral fusion with local autograft, at times mixed with bone marrow aspirate from the iliac crest.

\section{Data}

Demographics and comorbidities of all patients were collected by review of the electronic medical records. Demographics of interest included age (recorded in years), sex (male or female), and body mass index. Comorbidities of interest were the presence of hypertension, diabetes, heart disease, chronic obstructive pulmonary disease, kidney disease, liver disease, or hyperlipidemia. Smoking status was collected and recorded as nonsmokers, quit, or current. Current smoking was defined as smoking within 1 year of surgery. Nonsmokers were patients who never smoked. The rest of the patients were categorized as patients who quit smoking. Preoperative and postoperative pain and neurological status were recorded via the use of the neck pain visual analog scale (VAS) and Nurick scores, respectively. ${ }^{16}$ Preoperative and postoperative sagittal cervical Cobb angles were measured and recorded in degrees. We measured the global cervical Cobb angle by first drawing 2 lines: 1 parallel to the inferior endplate of the $\mathrm{C}-2$ vertebra and the other parallel to the superior endplate of the C-7 vertebra. Lines perpendicular to the first 2 lines were then drawn, and the angle from the intersecting perpendicular lines provided the angle of cervical curvature as previously described. ${ }^{18}$ A positive value indicated cervical lordosis, and a negative value indicated cervical kyphosis. Zero degrees indicated straightening of the spine and no curvature. The need for supplemental PSF was recorded.

The primary outcomes of interest were postoperative sagittal Cobb angle, adjacent-segment disease (ASD), neck pain VAS score, and Nurick score. ASD was defined as new disease affecting the level inferior and/or superior to the operated levels that required surgery. Secondary outcomes of interest included estimated blood loss (EBL), dysphagia, length of stay, perioperative complications, perioperative mortality, and pseudarthrosis rate. The EBL was recorded in milliliters and did not include blood loss from the PSF, if performed. Length of stay was defined as the number of days from surgery date to discharge date. Complication was defined as any unforeseen event requir- 

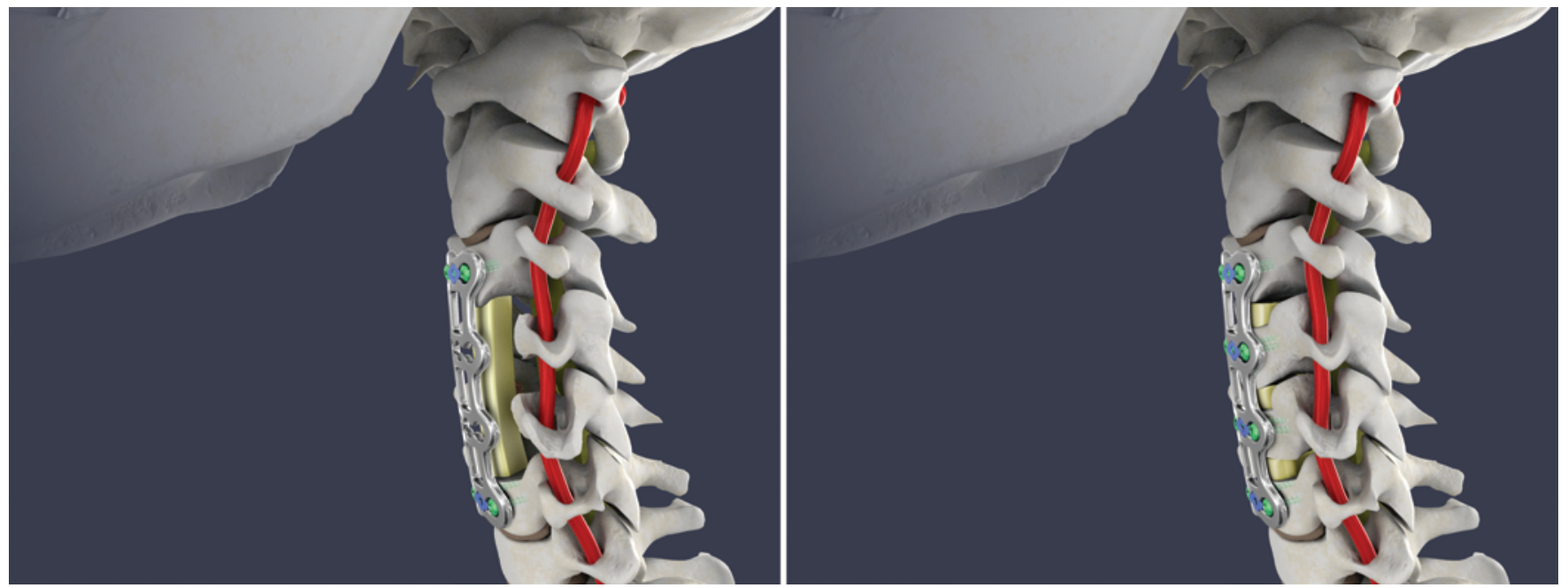

FIG. 1. Left: Artist illustration of 2-level ACCF at C3-6, with implantation of a single interbody cage and anterior plate fixation. Right: Artist illustration of 3-level ACDF at C3-6, with implantation of 3 separate interbody cages and anterior plate fixation.

ing additional medical and/or surgical intervention during the patient's hospitalization. Mortality was defined as death during hospital stay. All patients who were examined for pseudarthrosis were evaluated with dedicated 4-view radiographs of the cervical spine that included the lateral neutral, lateral flexion, lateral extension, and an anteriorposterior neutral view. Radiographic pseudarthrosis was defined as the presence of any of the following features: 1) radiolucent lines or absence of bridging trabecular bone across the fusion site; 2) presence of motion between the spinous processes on flexion-extension radiographs; or 3) presence of motion between vertebral bodies on flexionextension radiographs..$^{10,12}$

\section{Statistical Analysis}

Patients were separated into 2 groups: 2-level ACCF and 3-level ACDF. Descriptive statistics and comparisons among demographic groups, comorbidities, baseline clinical variables, perioperative outcomes, and follow-up outcomes were performed. A subgroup analysis of patients who did not undergo PSF was performed on outcomes that likely were influenced by PSF. A chi-square test was employed for categorical outcomes, and a 2-tailed Student t-test was performed for continuous outcomes. A p value $<$ 0.050 was used a threshold for statistical significance. All statistical analyses were performed with SAS 9.3 (SAS Institute).

\section{Results \\ Patients}

We identified 65 patients who underwent either 2-level ACCF or 3-level ACDF. Ten patients were then excluded because of diagnoses of tumor, trauma, or osteomyelitis. This resulted in a total of 55 patients who underwent surgery for CSM: 20 in the 2-level ACCF group and 35 in the 3-level ACDF group (Table 1). In the ACCF group, corpectomy was performed at the $\mathrm{C} 3-4$ levels in 1 patient, C4-5 levels in 4 patients, C5-6 levels in 11 patients, and
C6-7 levels in 4 patients. In the ACDF group, 20 patients underwent C4-7 ACDF, and 15 patients underwent C3-6 ACDF. Overall, 18 (32.7\%) underwent supplemental PSF (Table 2). Significantly more patients $(n=12,60.0 \%)$ underwent PSF in the ACCF group compared with patients $(\mathrm{n}=6,17.1 \%)$ who underwent $\operatorname{ACDF}(\mathrm{p}=0.001)$ (Table 2). Examples of patients who underwent 2-level ACCF for CSM can be seen in Fig. 2, and examples of patients who underwent 3-level ACDF for CSM appear in Fig. 3.

\section{Demographics, Comorbidities, and Preoperative Baseline}

Table 1 compares patients' demographics, comorbidities, and baseline clinical variables. Overall, the 2 groups were well matched regarding demographics and comorbidities. The mean age in the ACCF group was 54.8 years and in the ACDF group was 54.9 years. In the ACCF group, $65.0 \%$ were male, and in the ACDF group, $51.4 \%$ were male. There were no significant differences among any of the recorded comorbidities (hypertension, diabetes, heart disease, chronic obstructive pulmonary disease, kidney disease, liver disease, or hyperlipidemia) between the 2 groups. In addition, smoking status was similar. Ossification of the posterior longitudinal ligament was present in $4(7.3 \%)$ members of the whole cohort. There was no significant difference in the incidence of ossification of the posterior longitudinal ligament between patients who underwent 3-level ACCF and those who underwent 2-level ACDF (10.0\% vs 5.7\%, respectively, $\mathrm{p}=0.556)$. The mean preoperative neck pain VAS score among all patients studied was 4.8. There was no significant difference in neck pain VAS score between the 2 groups: 5.5 in the ACCF group versus 4.5 in the ACDF group $(p=0.414)$. The ACCF group had a significantly higher preoperative Nurick score (2.1) compared with the ACDF group (1.1) (p $=0.014)$. The overall mean preoperative sagittal cervical Cobb angle was $1.2^{\circ}$, and there were no significant differences between the ACCF and ACDF group $\left(-0.5^{\circ}\right.$ vs $2.5^{\circ}$, respectively, $\mathrm{p}=0.553$ ). 
TABLE 1. Comparison of demographics, comorbidities, and baseline clinical variables of patients who underwent 2-level ACCF vs 3-level ACDF

\begin{tabular}{|c|c|c|c|c|}
\hline Factor & Total $(n=55)$ & $\operatorname{ACCF}(n=20)$ & $\operatorname{ACDF}(n=35)$ & $\mathrm{p}$ Value \\
\hline Age (yrs) ${ }^{*}$ & $54.9 \pm 10.8$ & $54.8 \pm 12.9$ & $54.9 \pm 9.6$ & 0.957 \\
\hline Sex, no. of patients ( $\%$ male) & $31(56.4)$ & $13(65.0)$ & $18(51.4)$ & 0.389 \\
\hline Body mass index $\left(\mathrm{kg} / \mathrm{m}^{2}\right)^{*}$ & $31.6 \pm 33.5$ & $27.3 \pm 6.5$ & $34.2 \pm 41.9$ & 0.467 \\
\hline \multicolumn{5}{|l|}{ Comorbidity, no. of patients (\%) } \\
\hline Hypertension & $19(34.6)$ & $7(35.0)$ & $12(34.3)$ & 0.957 \\
\hline Diabetes & $5(9.1)$ & $3(15.0)$ & $2(5.7)$ & 0.249 \\
\hline Heart disease & $9(16.4)$ & $2(10.0)$ & $7(20.0)$ & 0.335 \\
\hline COPD & $1(1.8)$ & $0(0.0)$ & $1(2.9)$ & 0.446 \\
\hline Kidney disease & $6(10.9)$ & $3(15.0)$ & $3(8.6)$ & 0.462 \\
\hline Liver disease & $2(3.6)$ & $1(5.0)$ & $1(2.9)$ & 0.683 \\
\hline Hyperlipidemia & $12(21.8)$ & $3(15.0)$ & $9(25.7)$ & 0.355 \\
\hline Smoking, no. of patients (\%) & & & & 0.824 \\
\hline None & $20(36.4)$ & $7(35.0)$ & $13(37.1)$ & \\
\hline Quit & $22(40.0)$ & $9(45.0)$ & $13(37.1)$ & \\
\hline Current & $13(23.6)$ & $4(20.0)$ & $9(25.71)$ & \\
\hline Presence of OPLL, no. of patients (\%) & $4(7.3)$ & $2(10.0 \%)$ & $2(5.7 \%)$ & 0.556 \\
\hline Mean preop neck VAS score (range $0-10)^{*}$ & $4.8 \pm 3.4$ & $5.5 \pm 3.5$ & $4.5 \pm 3.3$ & 0.414 \\
\hline Mean preop Nurick score (range $0-5)^{\star}$ & $1.5 \pm 1.4$ & $2.1 \pm 1.5$ & $1.1 \pm 1.2$ & 0.014 \\
\hline Mean preop cervical Cobb angle $\left({ }^{\circ}\right)^{*}$ & $1.2 \pm 16.7$ & $-0.5 \pm 16.3$ & $2.5 \pm 17.3$ & 0.553 \\
\hline \multirow[t]{4}{*}{ Cervical levels (no. of patients) } & & C3-4 (1) & C4-7 (20) & NA \\
\hline & & C4-5(4) & C3-6 (15) & \\
\hline & & C5-6 (11) & & \\
\hline & & C6-7 (4) & & \\
\hline
\end{tabular}

COPD = chronic obstructive pulmonary disease; $\mathrm{NA}=$ not applicable; $\mathrm{OPLL}=$ ossification of posterior longitudinal ligament.

* Values expressed as the mean \pm standard deviation.

\section{Perioperative Outcomes}

Table 2 compares the perioperative outcomes between patients who underwent 2-level ACCF and 3-level ACDF. The ACCF group had significantly greater EBL when one compares only the anterior procedures $(\mathrm{p}<0.001)$ and longer length of stay $(\mathrm{p}=0.048)$ than the ACDF group. Overall EBL for the anterior stage of surgery was 230.5 $\mathrm{ml}$, and hospital stay was 5.7 days. The ACCF group had an EBL of $382.2 \mathrm{ml}$, and the ACDF group had an EBL of $117.9 \mathrm{ml}$. The ACCF group stayed in the hospital for a mean of 7.2 days, and the ACDF group stayed in the hospital for a mean of 4.9 days. Note that the ACCF group underwent significantly more second-stage PSF.

A total of $8(14.5 \%)$ of the 55 patients experienced complications (Table 2). There was a trend toward a higher number of complications among patients who underwent

TABLE 2. Comparison of perioperative outcomes of patients who underwent 2-level ACCF vs 3-level ACDF

\begin{tabular}{lcccc}
\hline \multicolumn{1}{c}{ Factor } & Total $(\mathrm{n}=55)$ & ACCF $(\mathrm{n}=20)$ & ACDF $(\mathrm{n}=35)$ & $\mathrm{p}$ Value \\
\hline $\mathrm{EBL}\left(\mathrm{cm}^{3}\right)^{*} \dagger$ & $230.5 \pm 212.1$ & $382.2 \pm 245.1$ & $117.9 \pm 69.0$ & $<0.001$ \\
\hline Mean length of stay (days) $\dagger$ & $5.7 \pm 4.2$ & $7.2 \pm 5.3$ & $4.9 \pm 3.3$ & 0.048 \\
\hline Complications (\%) & $8(14.5)$ & $4(20.0)$ & $2(5.7)$ & 0.102 \\
\hline Transient dysphagia, no. of patients (\%) & $5(9.1)$ & $2(10.0)$ & $3(8.6)$ & 0.860 \\
\hline Mortality rate, no. of patients (\%) & $0(0.0)$ & $0(0.0)$ & $0(0.0)$ & 0.999 \\
\hline Supplemental PSF, no. of patients (\%) & $18(32.7)$ & $12(60.0)$ & $6(17.1)$ & 0.001 \\
\hline Mean PSF levels (range) & $5.6(4-8)$ & $6.1(4-8)$ & $4.9(4-7)$ & 0.054 \\
\hline Posterior decompression, no. of patients (\%) & $10(55.6)$ & $5(41.7)$ & $5(83.3)$ & 0.094 \\
\hline
\end{tabular}

All analysis based on 18 patients who underwent PSF.

${ }^{*}$ Representing anterior only stage.

$\dagger$ Values expressed as the mean \pm standard deviation. 


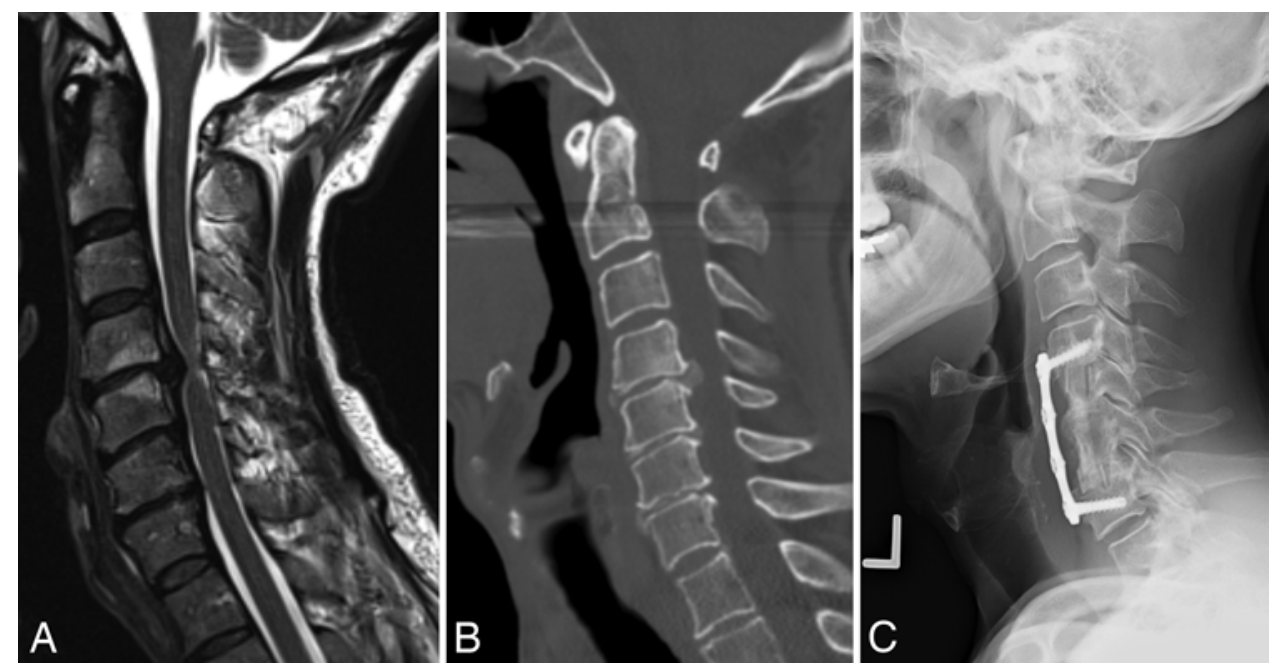

FIG. 2. Two-level ACCF for multilevel CSM. A: Sagittal T2-weighted MR image of the cervical spine showing severe spinal canal stenosis from C-4 to C-6 and focal spinal cord compression secondary to a herniated disc at the C4-5 level. B: Sagittal noncontrast CT scan of cervical spine showing a mildly calcified herniated disc and no evidence of ossification of the posterior longitudinal ligament. C: Lateral neutral cervical spine radiograph demonstrating C5-6 corpectomy, anterior plate fixation, and fusion.

ACCF $(20.0 \%)$ compared with patients who underwent $\mathrm{ACDF}(5.7 \%)$, but this was not statistically significant $(\mathrm{p}$ $=0.102)$; these complication rates included patients who underwent supplemental PSF. In the ACCF group, 1 patient experienced hardware failure, infection, and brachial plexus neuritis; 1 patient experienced a right C-5 palsy; 1 patient had an acute kidney injury; and 1 patient had pneumonia and a wound infection that required reoperation. In the ACDF group, 1 patient had dysphagia that required a feeding tube, and 1 patient had a wound infection that required antibiotics. Transient dysphagia (not requiring intervention) occurred in $5(9.1 \%)$ of the 55 patients. There was no significant difference in transient dysphagia rates between the ACCF and ACDF group (10.0\% vs $8.6 \%$, respectively, $\mathrm{p}=0.860)$. There were no perioperative deaths among patients in the cohort.

Among the 18 patients $(32.7 \%)$ who underwent supplemental PSF, a mean of 5.6 levels (range 4-8 levels) were fused and 10 patients $(55.6 \%)$ underwent posterior decompression. There was a trend for more levels to be fused in patients who underwent ACCF (mean 6.1 levels) compared to those who underwent ACDF (mean 4.9 levels) $(\mathrm{p}=0.054)$. However, there was a trend for more patients in the ACDF group to undergo posterior decompression compared with patients in the ACCF group $(83.3 \%$ vs $41.7 \%, \mathrm{p}=0.094)$.
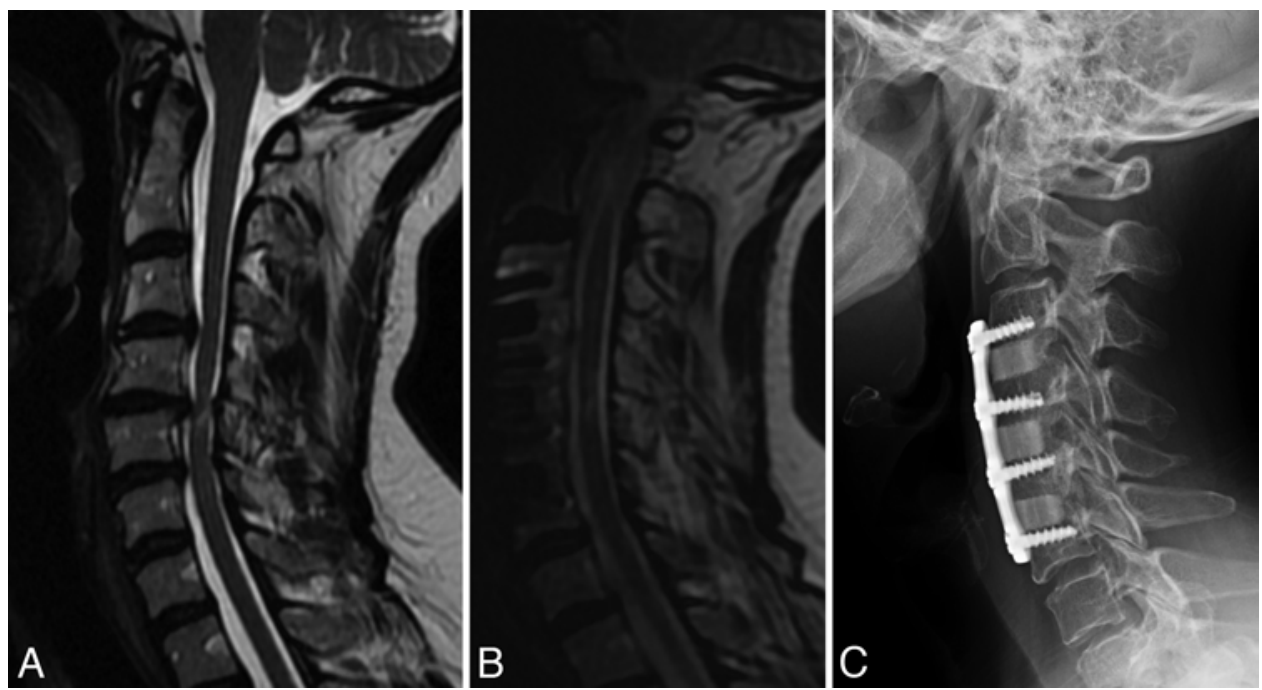

FIG. 3. Three-level ACDF for multilevel CSM. A: Sagittal T2-weighted MR image of the cervical spine showing severe to moderate spinal canal stenosis from the inferior endplate of $\mathrm{C}-3$ to the superior endplate of C-6. A T2 hyperintensity signal is seen within the cord at the level of C4-5. B: Sagittal T2-weighted MR image of the cervical spine following ACDF demonstrating adequate decompression with cerebrospinal fluid anterior and posterior to the cord. C: Lateral neutral cervical spine radiograph demonstrating discectomy decompression at C3-4, C4-5, and C5-6, anterior plate fixation, and fusion. 
TABLE 3. Comparison of postoperative and minimum 1-year follow-up outcomes of patients who underwent 2-level ACCF vs 3-level ACDF

\begin{tabular}{ccccc}
\hline Factor & Total $(\mathrm{n}=44)$ & ACCF $(\mathrm{n}=16)$ & ACDF $(\mathrm{n}=28)$ & $\mathrm{p}$ Value \\
\hline Postop Cobb angle $\left({ }^{\circ}\right)^{*} \dagger$ & $10.3 \pm 13.0$ & $7.2 \pm 13.9$ & $12.1 \pm 12.3$ & 0.173 \\
\hline Change in sagittal Cobb angle $\left({ }^{\circ}\right) \dagger$ & $9.3 \pm 9.2$ & $7.7 \pm 12.7$ & $10.3 \pm 7.0$ & 0.322 \\
\hline Length of follow-up (mos) $\dagger$ & $25.7 \pm 21.0$ & $32.1 \pm 25.2$ & $22.1 \pm 17.7$ & 0.127 \\
\hline Operative ASD, no. of patients (\%) & $2(4.5)$ & $1(6.3)$ & $1(3.6)$ & 0.682 \\
\hline Radiographic pseudarthrosis, no. of patients $(\%)$ & $3(6.8)$ & $1(6.3)$ & $2(7.1)$ & 0.909 \\
\hline Mean follow-up neck VAS score (range 0-10) ${ }^{*} \dagger$ & $1.3 \pm 2.4$ & $1.5 \pm 2.7$ & $1.2 \pm 2.3$ & 0.707 \\
\hline Change in neck VAS score (range 0-10) $\dagger$ & $3.2 \pm 3.5$ & $3.4 \pm 2.5$ & $3.2 \pm 3.8$ & 0.860 \\
\hline Mean follow-up Nurick score (range 0-5)* $\dagger$ & $0.8 \pm 1.4$ & $1.4 \pm 1.9$ & $0.4 \pm 1.0$ & 0.016 \\
\hline Change in Nurick score $\dagger$ & $0.73 \pm 1.1$ & $0.8 \pm 1.2$ & $0.7 \pm 1.0$ & 0.925 \\
\hline
\end{tabular}

Analysis including patient who under PSF.

* Significant change or improvement at postoperative or follow-up evaluation compared to preoperative evaluation $(p<0.001)$.

$\dagger$ Values expressed as the mean \pm standard deviation.

\section{Postoperative and Follow-Up Outcomes}

Table 3 compares postoperative and follow-up outcomes in patients who underwent 2-level ACCF and 3-level ACDF. Postoperatively, both groups obtained significant sagittal Cobb angle change compared with their preoperative Cobb angles $(\mathrm{p}<0.001)$ (Tables 1 and 3). Overall, the mean postoperative global cervical Cobb angle was $10.3^{\circ}$, $7.2^{\circ}$ in the ACCF group and $12.1^{\circ}$ in the ACDF group $(\mathrm{p}=$ 0.173). Changes in sagittal Cobb angle were similar as well: $7.7^{\circ}$ (gain of lordosis) in ACCF group and $10.3^{\circ}$ (gain of lordosis) in the ACDF group ( $\mathrm{p}=0.322)$.

Of the 55 patients, a total of $44(80.0 \%)$ were available for follow-up at least 1 year after surgery (Table 3). Followup ranged from 12 to 69 months. Mean follow-up duration in patients who underwent ACCF was 32.1 months, and mean follow-up duration in the ACDF group was 22.1 months. Symptomatic ASD that required surgery occurred
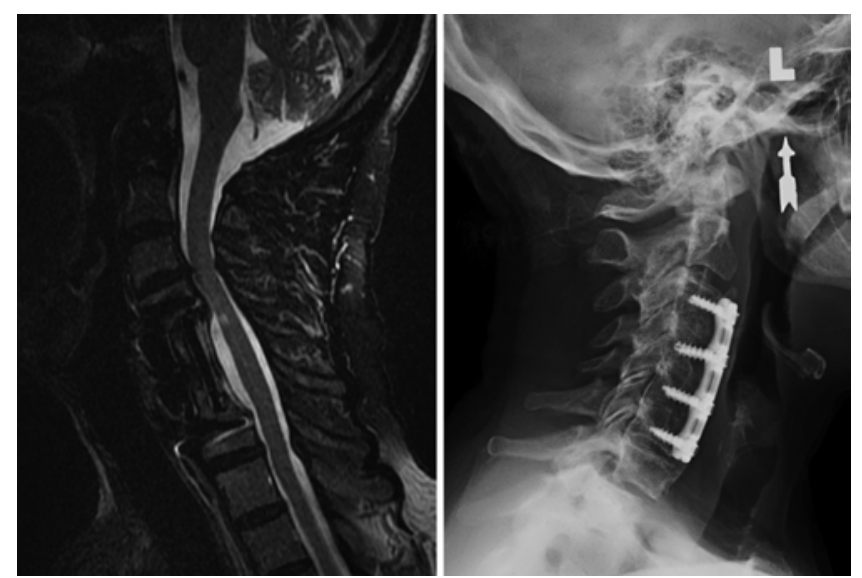

FIG. 4. ASD following 2-level ACCF and 3-level ACDF. Left: Sagittal T2-weighted MR image of the cervical spine demonstrating prior C5-6 ACCF and ASD in the form of severe spinal canal stenosis at C3-4. Right: Lateral neutral cervical spine radiograph showing prior C3-4, C4-5, and C5-6 ACDF and ASD in the form of a degenerative disc and loss of disc height at the C6-7 disc space. In addition, there is new anterolisthesis of $\mathrm{C}-7$ relative to $\mathrm{T}-1$. in 2 patients (4.5\%), and there was no significant difference in the ASD rate between the 2 groups: $1(6.3 \%)$ in the ACCF group and $1(3.6 \%)$ in the ACDF group $(p=0.682)$. Both groups included patients who underwent surgery for ASD (Fig. 4). The overall radiographic pseudarthrosis rate was $6.8 \%$. There was no significant difference in the rate of pseudarthrosis between patients who underwent ACCF $(6.3 \%)$ and those who underwent ACDF (7.1\%) $(\mathrm{p}=0.909)$. Examples of pseudarthrosis following ACCF can be seen in Fig. 5A, and examples following ACDF can be seen in Fig. 5B and C.

Both groups obtained significant benefit from surgery in terms of Nurick scores $(\mathrm{p}<0.001)$ and neck pain VAS scores $(\mathrm{p}<0.001)$ at follow-up compared with their preoperative evaluations. Similar magnitudes of neck pain VAS score improvements were seen in both ACCF and ACDF groups at follow-up (3.4 vs 3.2, respectively; $p=0.860$ ) (Table 3 ), and neck pain VAS scores were similar (1.5 vs $1.2, p=0.707)$ (Table 3$)$. Both groups obtained similar improvements in Nurick scores (0.8 vs 0.7, $\mathrm{p}=0.925)$ (Table 3 ), but there remained a significant difference in Nurick scores between the 2 groups at follow-up (1.4 vs $0.4, \mathrm{p}=$ $0.016)$.

\section{Subgroup Analysis: Comparison Without Posterior Spinal Fusion}

Table 4 shows the results of subgroup comparisons of patients who underwent 2-level ACCF and 3-level ACDF without PSF. Results were available for 8 patients in the ACCF group and 29 patients in the ACDF group. Analysis showed no significant difference in length of hospital stay between the 2-level ACCF group and the 3-level ACDF group (3.1 days vs 4.4 days, respectively, $\mathrm{p}=0.267$ ). In addition, there was no longer a trend for a higher complication rate in patients who underwent 2-level ACCF compared with those who underwent 3-level ACDF $(0.0 \%$ vs $3.4 \%$, respectively, $\mathrm{p}=0.594$ ). Regarding postoperative and follow-up outcomes, similar associations and findings were seen among the whole cohort and the ACCF and ACDF subgroups (Table 4). Specifically, there was no significant difference in operative $\operatorname{ASD}(12.5 \%$ vs $4.2 \%, \mathrm{p}=$ 


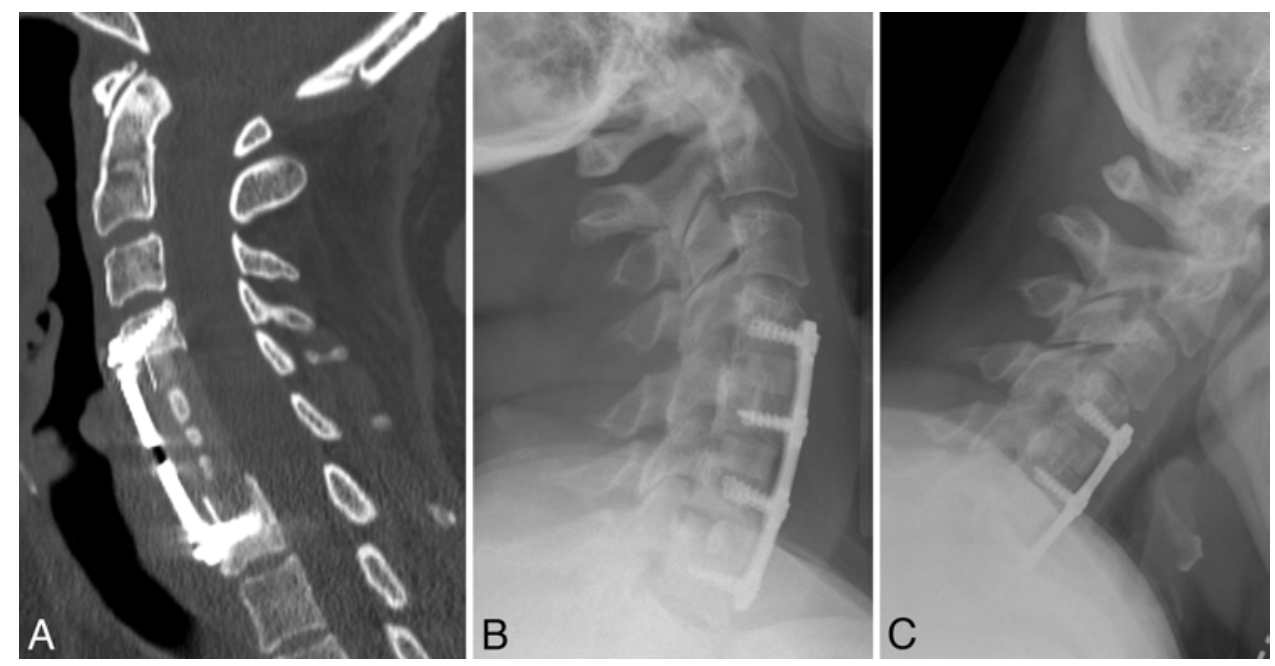

FIG. 5. Pseudarthrosis following 2-level ACCF and 3-level ACDF. Sagittal CT scan of the cervical spine (A) demonstrating prior C5-6 corpectomy and lack of bony fusion within the corpectomy site. Lateral extension (B) and flexion (C) radiographs of the cervical spine showing prior $\mathrm{C} 4-5, \mathrm{C} 5-6$, and C6-7 ACDF and hyperdynamic movement (changes in distance between the spinous processes) during flexion and extension. A lucency surrounds the screw head at the C-6 level.

$0.399)$ and radiographic pseudarthrosis $(12.9 \%$ vs $6.9 \%, \mathrm{p}$ $=0.607$ ) between patients who underwent 2-level ACCF compared to 3-level ACDF, respectively.

\section{Discussion}

Only a few studies have directly addressed the controversy regarding the superiority of ACCF versus ACDF for the treatment of multilevel CSM. Most have either compared groups with different numbers of operated levels or have strictly concentrated on comparing l-level ACCF versus 2-level ACDF (i.e., comparing equivalent numbers of disc segments). In addition, the studies that do compare 1-level ACCF versus 2-level ACDF consist of relatively small cohorts. ${ }^{17,19,25}$ In 2001, Wang et al. published a retrospective study that examined clinical and radiographic outcomes of 52 consecutive patients who underwent anterior decompression: 20 patients underwent 1-level ACCF, and 32 patients underwent 2-level ACDF. ${ }^{25}$ The authors did not find significant differences in pseudarthrosis rates, extents of graft subsidence, or clinical outcomes (based on Odom's criteria). Oh et al. performed a similar retrospective study of 31 patients and compared 1-level ACCF and 2-level ACDF. ${ }^{17}$ However, in addition to evaluating radiological and clinical outcomes, they also compared perioperative outcomes and found that ACCF was associated with significantly longer operative time (210 minutes vs 140 minutes), greater blood loss $\left(779 \mathrm{~cm}^{3}\right.$ vs $\left.306 \mathrm{~cm}^{3}\right)$, lower segmental height $\left(5.0^{\circ}\right.$ vs $\left.5.6^{\circ}\right)$, and lower postoperative cervical lordosis $\left(14.6^{\circ}\right.$ vs $\left.23.4^{\circ}\right)$. But as was the case in the study by Wang et al., there was no difference in

TABLE 4. Subgroup comparisons of patients who underwent 2-level ACCF vs 3-level ACDF without PSF

\begin{tabular}{|c|c|c|c|c|}
\hline Factor & Total & ACCF & ACDF & $\mathrm{p}$ Value \\
\hline \multicolumn{5}{|l|}{ Periop outcomes* } \\
\hline Length of stay (days) $\dagger$ & $4.2 \pm 2.9$ & $3.1 \pm 1.0$ & $4.4 \pm 3.2$ & 0.267 \\
\hline Complications (\%) & $1(2.7)$ & $0(0.0)$ & $1(3.4)$ & 0.594 \\
\hline \multicolumn{5}{|l|}{ Postop \& follow-up outcomesł } \\
\hline Postop Cobb angle $\left({ }^{\circ}\right) \dagger$ & $10.9 \pm 10.2$ & $9.0 \pm 6.3$ & $11.4 \pm 11.1$ & 0.567 \\
\hline Cobb angle change $\left({ }^{\circ}\right) \dagger$ & $9.9 \pm 5.0$ & $9.3 \pm 6.0$ & $10.1 \pm 4.8$ & 0.688 \\
\hline Operative ASD, no. of patients (\%) & $2(6.3)$ & $1(12.5)$ & $1(4.2)$ & 0.399 \\
\hline Radiographic pseudarthrosis, no. of patients (\%) & $3(8.1)$ & $1(12.5)$ & $2(6.9)$ & 0.607 \\
\hline Follow-up neck VAS score $(0-10) \dagger$ & $1.5 \pm 2.6$ & $2.0 \pm 3.2$ & $1.4 \pm 2.5$ & 0.558 \\
\hline Change in neck VAS score $(0-10) \dagger$ & $3.3 \pm 3.7$ & $3.6 \pm 2.4$ & $3.3 \pm 4.0$ & 0.870 \\
\hline Follow-up Nurick score $(0-5) \dagger$ & $0.3 \pm 0.8$ & $0.3 \pm 0.5$ & $0.3 \pm 0.8$ & 0.789 \\
\hline Change in Nurick score $†$ & $1.0 \pm 1.0$ & $1.4 \pm 0.5$ & $0.8 \pm 1.1$ & 0.105 \\
\hline
\end{tabular}

* Based on data obtained in 37 patients overall (8 in the ACCF group and 29 in the ACDF group).

$\dagger$ Values are expressed as the mean \pm standard deviation.

$\ddagger$ Based on data obtained in 32 patients overall ( 8 in the ACCF group and 24 in the ACDF group). 
pseudarthrosis rate or clinical outcome (based on the Japanese Orthopaedic Association score and pain). Following these 2 studies, Park et al. performed a large retrospective review of prospectively collected data comparing outcomes of 52 patients who underwent 1-level ACCF versus 45 patients who underwent 2-level ACDF for CSM. ${ }^{19}$ Their findings after at least 1 year of follow-up suggested that the 2 procedures yielded comparable results in terms of sagittal alignment, cervical lordosis, graft subsidence, and adjacent-level ossification. Taken together, it seems from these studies that 2-level ACDF was associated with less blood loss and shorter operative times than 1-level ACCF. However, long-term clinical outcomes were similar, and radiological findings were mixed.

More often than not, CSM involves multiple disc and vertebral levels. However, surgeons continue to lack data and studies that compare the outcomes of ACCF and ACDF. Often, the outcomes following ACCF versus ACDF for multilevel CSM that spans 3 or more disc segments are mixed into larger cohorts with different numbers of operated levels, and the patients of interest typically make up only a small portion of the evaluated cohort. ${ }^{1,8,11,13,20,23}$ Similarly, in their reported criteria for article selection, recent systematic reviews and meta-analyses that compare ACCF versus ACDF for multilevel CSM do not specify or account for the number of disc segments treated and thus review articles with mixed cohorts. ${ }^{7,9}$ Without a subgroup analysis, the findings from such studies are likely skewed by a heterogeneous cohort and may not be directly applicable in the clinical setting. Therefore, the goal of the present study was to compare 2-level ACCF versus 3-level ACDF for multilevel CSM.

Two retrospective studies published by Guo et al. and Liu et al. (from the same department and institution) performed a comparative study of 3 anterior decompressive and fusion techniques specifically for CSM that spanned 3 disc segments. ${ }^{6,14}$ Both studies compared similar perioperative and radiological outcomes following ACDF alone versus hybrid $\mathrm{ACDF}-\mathrm{ACCF}$ versus $\mathrm{ACCF}$ alone. The 2 studies differed mainly by evaluating patients during different time periods. Guo et al. evaluated 120 patients from 2006 to 2008, and Liu et al. identified an additional 60 patients (totaling 180 patients) by encompassing a longer time span from 2003 to 2010 . They each performed a subgroup analysis that compared 2-level ACCF and 3-level ACDF. Guo et al. included 24 patients in the ACCF group and 43 patients in the ACDF group. Liu et al. included 69 patients in the ACDF group and 39 patients in the ACCF group. The 2 studies found that 3-level ACDF involved shorter operative time, less blood loss, lower complication rates, and greater cervical lordosis compared with 2-level ACCF. In our study, 3-level ACDF was associated with less blood loss compared with 2-level ACCF, but we found no differences in complication rates and postoperative cervical lordosis.

Among the published reports, most evidence suggests that 2-level ACCF is associated with greater blood loss than is 3-level ACDF, and this also agrees with the findings from our study ${ }^{6,13,14,20}$ Although multiple studies have shown increases in blood loss associated with ACCF, the clinical significance is questionable because the amount of additional blood loss is relatively small (ranging from an additional $46 \mathrm{ml}$ to $273 \mathrm{ml}$ ). This volume usually does not warrant blood transfusions.

Longer hospital stays can have important clinical implications because they represent time to recovery, increase the risk for nosocomial complications, and increase the cost of medical care. In the few studies that have addressed this issue, data suggest that 2-level ACCF is associated with longer hospital stays than is 3-level ACDF. ${ }^{8,20}$ An additional 8 days of hospital stay has been reported in a previous study. ${ }^{20}$ We also found that patients who underwent ACCF stayed in the hospital approximately 2 days longer than patients who underwent ACDF, but the ACCF group had a significantly larger proportion of patients who underwent second-stage PSF. A subgroup analysis of patients who did not undergo PSF showed no significant difference in length of stay. Most studies that compare ACCF versus ACDF for multilevel CSM do not report or address the utilization of PSF. ${ }^{6,8,13,14,20}$ This is a major confounder because a staged procedure days after the initial ACDF or ACCF predictably lengthens a hospital stay.

Although staged PSF is one of the major influences on hospital stay, longer hospital stays observed in patients who underwent ACCF may also be secondary to more complications: The 2-level ACCF group experienced approximately $15 \%$ more complications than did the ACDF group (Table 2). These results, however, occurred in the context of more ACCF patients undergoing PSF. A subgroup comparison that excluded PSF patients no longer found a trend toward greater complication rates or differences in complication rates between the 2 groups. Findings from previous studies regarding complication rates are mixed. Some reports suggested that 2-level ACCF is associated with complication rates that are as much as $22 \%$ higher than that of ACDF ${ }^{6,14}$ Other studies, however, have shown no difference in complication rates between ACCF and $A C D F{ }^{13,20}$ However, one must emphasize again that drawing conclusions from these studies is limited because they do not specifically address the utilization of PSF. According to the findings from the present study, it seems that length of stay and complications are similar between groups when only anterior decompression is performed.

All patients experienced significant improvements in cervical lordosis after surgery. The radiographic findings of improved cervical lordosis trended toward a higher value in patients who underwent 3-level ACDF compared with 2-level ACCF, but the difference was not statistically significant. Most studies have shown greater cervical lordosis postoperatively in patients who underwent ACDF compared with those who underwent ACCF; typical differences were approximately $3-12^{\circ} \cdot{ }^{6,13,14,20}$ Biomechanically, distraction at multiple points (disc spaces) during ACDF allows more effective curvature correction and change in lordosis. In the case of 3-level ACDF, there are effectively 3 areas of distraction, and in 2-level ACCF, there is effectively 1 area of distraction. The opportunity to correct for normal lordosis should be pursued when possible. Clinical studies have directly correlated the presence of cervical kyphosis following surgery with greater neurological disability and pain, and patients who obtain lordosis after surgery have better functional outcomes. ${ }^{5}$ 
Regarding radiographic pseudarthrosis rates, outcomes were similar for both groups. One of the key initial controversies regarding whether ACCF or ACDF should be used in the treatment of CSM stems from older reports (from almost 20 years ago) of low fusion rates associated with multilevel ACDF. In the past, pseudarthrosis rates as high as $48 \%$ have been reported for 3-level ACDF. ${ }^{21}$ The theory regarding such high pseudarthrosis rates is based on the fact that greater numbers of graft-host interfaces are present when multilevel ACDF is performed compared with ACCF. However, according to more recent studies and the findings from the current study, significant differences in pseudarthrosis rates no longer seem to be the case. Both groups in this study had similar rates of pseudarthrosis. Similarly, studies by Song el al., Hwang et al., and Guo et al. showed similar fusion rates between patients who underwent multilevel ACCF and ACDF for CSM, and their rates of fusion ranged from $88 \%$ to $97 \%{ }^{6,8,20}$ In fact, data suggest that 3-level ACDF may actually offer higher rates of fusion than 2-level ACCF (100\% vs $85 \%-90 \%$, respectively). ${ }^{13,14}$ The recent reports of low pseudarthrosis rates may represent outcomes following the utilization of more advanced techniques, equipment, and technology.

Overall, operative ASD occurred in approximately $4.5 \%$ of the cohort $(6.3 \%$ in $\mathrm{ACCF}$ group and $3.6 \%$ in ACDF group) (Table 3 ), which is similar to the previously reported incidence of ASD requiring reoperation. ${ }^{3}$ Liu et al. identified ASD based strictly on radiological findings despite the presence of clinical symptomology, and therefore they reported a high ASD rate of $51.9 \%$ following anterior decompression and fusion for 3-level CSM. ${ }^{14}$ Despite the differences in the definition of ASD, their findings were similar because they found no significant differences in ASD between patients who underwent 2-level ACCF and those who underwent 3-level ACDF. This remained consistent even in patients who did not undergo PSF.

The main limitations to this study relate to its retrospective nature and the cohort size. The retrospective design places this study at risk for confounders, including the worse Nurick scores and higher rates of PSF in the ACCF group. The 2 cohorts in our study were well matched in terms of demographics and comorbidities. But the ACCF group had a significantly higher mean preoperative Nurick score compared with the ACDF group, and the Nurick score in the ACCF group continued to remain higher postoperatively. Despite this difference, the most important observation was that both groups had similar magnitudes of improvement. In addition, 2-level ACCF and 3-level ACDF were both effective in providing similar neck pain relief. Among published reports, authors seem to agree that both $\mathrm{ACCF}$ and $\mathrm{ACDF}$ are able to provide adequate and similar benefits in terms of long-term neurological outcomes and pain relief in the treatment of multilevel CSM. ${ }^{6,8,13,14,20}$ One should bear in mind that our outcomes were obtained with the utilization of PSF in a majority of the patients who underwent ACCF. The use of PSF is secondary to customs in clinical practice and cannot be readily controlled in retrospective studies, but this was addressed in a subgroup analysis. Further, one should recall that after we excluded patients with PSF, more patients in the ACDF group than in the ACCF group were available for subgroup analysis.
Despite the drawbacks, this study consists of a cohort that can be generalized to surgical candidates with multilevel CSM who are undergoing evaluation for either 2-level ACCF or 3-level ACDF.

Because there continues to be a need for additional research regarding multilevel ACCF and ACDF, studies that concentrate on the costs associated with the 2 procedures would be valuable. Specifically, a direct-cost and costeffectiveness analysis based on quality of life outcomes comparing the 2 procedures would assist in the selection of a procedure for SCM. Unfortunately, no currently published studies evaluate this outcome.

\section{Conclusions}

According to the findings from this study, 2-level ACCF for multilevel CSM was associated with higher EBL and longer hospital stays when patients underwent a secondstage PSF. Otherwise, the length of stay was similar when patients underwent anterior decompression without PSF. Similarly, perioperative complication rates trended to be higher when patients underwent supplemental PSF but not after anterior decompression without PSF. Both groups obtained similar postoperative cervical lordosis, operative ASD rates, radiographic pseudarthrosis rates, neurological improvement, and pain relief. Therefore, except for blood loss, both 2-level ACCF and 3-level ACDF provided similar outcomes in the treatment of multilevel CSM when PSF was not required. The intricacies in deciding when to use ACDF or ACCF depend on multiple factors and can be complex. Anatomical and pathological differences, the presence of morbidity, and bone quality among patients should be taken into account during preoperative evaluation. In addition, because both approaches provide relatively similar outcomes, future cost-analysis studies are needed to determine which procedure is more cost effective based on quality of life outcomes.

\section{References}

1. Burkhardt JK, Mannion AF, Marbacher S, Dolp PA, Fekete TF, Jeszenszky D, et al: A comparative effectiveness study of patient-rated and radiographic outcome after 2 types of decompression with fusion for spondylotic myelopathy: anterior cervical discectomy versus corpectomy. Neurosurg Focus 35(1):E4, 2013

2. Fountas KN, Kapsalaki EZ, Nikolakakos LG, Smisson HF, Johnston KW, Grigorian AA, et al: Anterior cervical discectomy and fusion associated complications. Spine (Phila Pa 1976) 32:2310-2317, 2007

3. Gao R, Yang L, Chen H, Liu Y, Liang L, Yuan W: Long term results of anterior corpectomy and fusion for cervical spondylotic myelopathy. PLoS ONE 7:e34811, 2012

4. Gore DR: The arthrodesis rate in multilevel anterior cervical fusions using autogenous fibula. Spine (Phila Pa 1976) 26:1259-1263, 2001

5. Grosso MJ, Hwang R, Mroz T, Benzel E, Steinmetz MP: Relationship between degree of focal kyphosis correction and neurological outcomes for patients undergoing cervical deformity correction surgery. J Neurosurg Spine 18:537-544, 2013

6. Guo Q, Bi X, Ni B, Lu X, Chen J, Yang J, et al: Outcomes of three anterior decompression and fusion techniques in the treatment of three-level cervical spondylosis. Eur Spine J 20:1539-1544, 2011 
7. Han YC, Liu ZQ, Wang SJ, Li LJ, Tan J: Is anterior cervical discectomy and fusion superior to corpectomy and fusion for treatment of multilevel cervical spondylotic myelopathy? A systemic review and meta-analysis. PLoS ONE 9:e87191, 2014

8. Hwang SL, Lee KS, Su YF, Kuo TH, Lieu AS, Lin CL, et al: Anterior corpectomy with iliac bone fusion or discectomy with interbody titanium cage fusion for multilevel cervical degenerated disc disease. J Spinal Disord Tech 20:565-570, 2007

9. Jiang SD, Jiang LS, Dai LY: Anterior cervical discectomy and fusion versus anterior cervical corpectomy and fusion for multilevel cervical spondylosis: a systematic review. Arch Orthop Trauma Surg 132:155-161, 2012

10. Kaiser MG, Mummaneni PV, Matz PG, Anderson PA, Groff MW, Heary RF, et al: Radiographic assessment of cervical subaxial fusion. J Neurosurg Spine 11:221-227, 2009

11. Li J, Zheng Q, Guo X, Zeng X, Zou Z, Liu Y, et al: Anterior surgical options for the treatment of cervical spondylotic myelopathy in a long-term follow-up study. Arch Orthop Trauma Surg 133:745-751, 2013

12. Lin Q, Wang X, Zhou X, Chen H, Shen X, Yuan W, et al: A comparison of the Gallie technique and casting versus the harms technique for the treatment of odontoid fractures. $\mathbf{J}$ Orthop Trauma 25:670-673, 2011

13. Lin Q, Zhou X, Wang X, Cao P, Tsai N, Yuan W: A comparison of anterior cervical discectomy and corpectomy in patients with multilevel cervical spondylotic myelopathy. Eur Spine J 21:474-481, 2012

14. Liu Y, Hou Y, Yang L, Chen H, Wang X, Wu X, et al: Comparison of 3 reconstructive techniques in the surgical management of multilevel cervical spondylotic myelopathy. Spine (Phila Pa 1976) 37:E1450-E1458, 2012

15. Mummaneni PV, Kaiser MG, Matz PG, Anderson PA, Groff MW, Heary RF, et al: Cervical surgical techniques for the treatment of cervical spondylotic myelopathy. J Neurosurg Spine 11:130-141, 2009

16. Nurick S: The pathogenesis of the spinal cord disorder associated with cervical spondylosis. Brain 95:87-100, 1972

17. Oh MC, Zhang HY, Park JY, Kim KS: Two-level anterior cervical discectomy versus one-level corpectomy in cervical spondylotic myelopathy. Spine (Phila Pa 1976) 34:692-696, 2009

18. Ohara A, Miyamoto K, Naganawa T, Matsumoto K, Shimizu $\mathrm{K}$ : Reliabilities of and correlations among five standard methods of assessing the sagittal alignment of the cervical spine. Spine (Phila Pa 1976) 31:2585-2592, 2006
19. Park Y, Maeda T, Cho W, Riew KD: Comparison of anterior cervical fusion after two-level discectomy or single-level corpectomy: sagittal alignment, cervical lordosis, graft collapse, and adjacent-level ossification. Spine J 10:193-199, 2010

20. Song KJ, Lee KB, Song JH: Efficacy of multilevel anterior cervical discectomy and fusion versus corpectomy and fusion for multilevel cervical spondylotic myelopathy: a minimum 5-year follow-up study. Eur Spine J 21:1551-1557, 2012

21. Swank ML, Lowery GL, Bhat AL, McDonough RF: Anterior cervical allograft arthrodesis and instrumentation: multilevel interbody grafting or strut graft reconstruction. Eur Spine J 6:138-143, 1997

22. Toledano M, Bartleson JD: Cervical spondylotic myelopathy. Neurol Clin 31:287-305, 2013

23. Uribe JS, Sangala JR, Duckworth EA, Vale FL: Comparison between anterior cervical discectomy fusion and cervical corpectomy fusion using titanium cages for reconstruction: analysis of outcome and long-term follow-up. Eur Spine J 18:654-662, 2009

24. Wang JC, Hart RA, Emery SE, Bohlman HH: Graft migration or displacement after multilevel cervical corpectomy and strut grafting. Spine (Phila Pa 1976) 28:1016-1022, 2003

25. Wang JC, McDonough PW, Endow KK, Delamarter RB: A comparison of fusion rates between single-level cervical corpectomy and two-level discectomy and fusion. J Spinal Disord 14:222-225, 2001

26. Young WF: Cervical spondylotic myelopathy: a common cause of spinal cord dysfunction in older persons. Am Fam Physician 62:1064-1070, 1073, 2000

\section{Author Contributions}

Conception and design: all authors. Acquisition of data: Lau. Analysis and interpretation of data: Mummaneni, Lau. Drafting the article: Lau. Critically revising the article: all authors. Reviewed submitted version of manuscript: all authors. Approved the final version of the manuscript on behalf of all authors: Mummaneni. Statistical analysis: Lau. Study supervision: Mummaneni.

\section{Correspondence}

Praveen V. Mummaneni, Department of Neurological Surgery, University of California, San Francisco, 505 Parnassus Ave., Rm. M779, San Francisco, CA 94143-0112.email: mummanenip@ neurosurg.ucsf.edu. 